\title{
MOTIVATION ET PRÉPARATION A LA PERFORMANCE SPORTIVE
}

\section{Christophe GERNIGON}

Laboratoire de Psychologie Appliquée au Sport, INSEP, Paris.

Gernigon, C. (1998). Motivation et préparation à la performance sportive. In P. Fleurance (Ed.), Entraînement mental et sport de haute performance (pp. 121-163). Paris: INSEP Publications.

MOTS CLES : Motivation, perceptions de contrôle, sentiment de compétence, préparation mentale, performance.

Il est communément admis que pour atteindre un haut niveau de performance, quel que soit le domaine concerné, une très forte motivation est requise. Le contexte de la performance sportive n'échappe pas à cette règle. L'accès et le maintien d'un athlète parmi l'élite s'affranchit difficilement d'un engagement total de celui-ci dans la pratique de son sport. Aussi n'oserait-on imaginer un seul instant qu'un champion puisse ne pas être motivé par son activité. Pourtant, ce serait oublier un peu vite qu'accepter la pénibilité et la répétitivité de certains entraînements, sacrifier une partie de sa vie familiale, professionnelle ou ses loisirs, subir les conséquences des blessures et des échecs constituent autant de facteurs de découragement qui peuvent compromettre gravement la réussite d'une saison voire d'une carrière sportive. Devant le caractère capital que revêt le développement et l'entretien de la motivation dans la préparation à la performance, il apparaît nécessaire d'identifier précisément les processus motivationnels sollicités par les comportements les plus persévérants ainsi que les facteurs à l'origine de ses processus. Une telle identification devrait permettre ensuite de dégager les principes susceptibles de guider l'intervention de ceux qui ont en charge d'accompagner l'athlète et de l'aider à se maintenir au plus haut niveau.

\section{1 - QU'EST-CE QUE LA MOTIVATION CHEZ LE SPORTIF?}

D'une manière générale, la motivation peut être définie succinctement comme " l'aspect dynamique et directionnel (sélectif ou préférentiel) du comportement " (Nuttin, 1985). Pour Maehr (1984), l'opérationalisation de cette double dimensionalité se manifeste chez un individu par la direction de son attention et de son activité, par la persistance dans cette dernière (spécialement face aux obstacles), par l'intensité de son activité (efforts), par la continuité de cette activité en dehors de toute contrainte externe et enfin par ses performances. Toutefois, ce dernier indicateur représente davantage la conséquence des précédents. Il est probable, en effet, qu'une personne motivée (donc qui s'attache intensément, 
avec persévérance et librement à tenter de réussir une tâche choisie) augmente ses chances d'obtenir de bons résultats (Stipek, 1993). Mais il ne s'agit là que d'une probabilité tant il est vrai, notamment en sport, que des comportements motivés peuvent malheureusement être suivis d'un échec.

Plusieurs registres motivationnels peuvent être impliqués dans la pratique sportive (Durand, 1987 ; Thill, 1989). Si l'engagement d'un sportif peut répondre à une recherche directe de la sensation hédonique que procure son activité ou bien encore à un besoin d'affiliation, son accession et son maintien au plus haut niveau de la compétition relèvent davantage d'une motivation d'accomplissement. Pour ses premiers théoriciens (Atkinson, 1957 ; McClelland, Atkinson, Clark, \& Lowell, 1953 ; Murray, 1938), la motivation d'accomplissement se caractérise par les comportements que les personnes manifestent lorsqu'elles s'appliquent à bien réaliser une tâche, à rechercher des normes d'exigence ou de performance, à se fixer des buts élevés. Cette définition semble correspondre parfaitement aux caractéristiques de la pratique sportive d'élite. Dans une même logique, on peut assimiler le sport de haut niveau à un contexte d'accomplissement tel que le définit Heckhausen (1974). Pour cet auteur, plusieurs conditions doivent en effet être réunies pour que l'on puisse évoquer un contexte d'accomplissement :

1) Tout d'abord les tâches à réaliser dans ce contexte doivent être finalisées par un but objectif et identifiable. C'est le cas dans les activités sportives, à tel point que l'atteinte des buts fait l'objet d'évaluations en termes de performances.

2) Il doit être possible d'estimer la quantité d'effort nécessaire pour être performant, ce qui suppose également de la part de celui qui est engagé dans la tâche une estimation de sa propre compétence au regard de la difficulté de cette tâche. Les activités sportives fournissent de multiples opportunités pour un sportif de tester ses compétences et d'évaluer les efforts nécessaires pour obtenir des résultats, efforts qu'il peut d'ailleurs juger inutiles compte tenu de la difficulté perçue (Kukla, 1972) et de ses buts personnels (Nicholls, 1984a, 1984b).

3) Les comportements manifestés par les individus doivent pouvoir aboutir à l'échec comme au succès. Il est malheureusement aisé de montrer que l'incertitude des résultats en sport est une réalité bien présente aux conséquences parfois dommageables.

4) La quantité d'efforts consentie ainsi que l'estimation de la difficulté doivent répondre à des standards d'excellence. Ces standards peuvent être sociaux, personnels ou objectifs par la référence aux autres, à ses propres performances antérieures ou bien à la réalisation d'une tâche. Là encore, les activités sportives offrent de nombreuses occasions d'étalonner ces standards selon chacune de ces trois manières, même si la compétition privilégie la référence aux standards sociaux.

5) Les performances réalisées par un individu doivent être, dans une large mesure, le résultat de ses propres actions, même si des facteurs extérieurs au sujet peuvent également intervenir. Le sportif est responsable de ses résultats même si parfois, dans certaines activités, 
cette responsabilité est en partie partagée par des partenaires et/ou des adversaires ou encore certains facteurs environnementaux (arbitrage, public, etc.).

Par la présence de toutes ces caractéristiques dont le rôle est exacerbé par les enjeux médiatiques, financiers voire politiques que la compétition moderne revêt, le contexte sportif sollicite et détermine intensément la motivation d'accomplissement de l'athlète. Cette motivation entretient de multiples relations avec sa performance en intervenant à tous les moments de la vie sportive : pendant la compétition mais aussi après celle-ci, ce qui constitue déjà les prémisses de la prochaine. Les répercussions sur la performance peuvent être directes et ponctuelles par la régulation de l'activation (Sanders, 1983), de l'attention (Carver \& Scheier, 1981 ; Duval \& Wicklund, 1972; White \& Duda, 1991) et de l'anxiété (Duda, Newton, \& Chi, 1990 ; Hall \& Kerr, 1997 ; Nicholls, 1989 ; Vealey \& Campbell, 1988) que les états motivationnels autorisent. Mais la motivation agit surtout comme processus continu indirectement impliqué dans la performance. Ses effets se manifestent alors sur la pertinence des objectifs choisis par le sportif, l'intensité de ses efforts et sa persévérance à l'entraînement ainsi que sur l'adhésion à un style de vie compatible avec les exigences de la haute compétition (sacrifices familiaux, professionnels, ascèse, etc.). Ces variables dépendantes de la motivation d'accomplissement sont donc responsables de la qualité de la préparation physique, mentale, technique et tactique du sportif. La qualité de cette préparation détermine à son tour les niveaux d'habileté acquis dans chacun de ses registres.

A l'origine de tous les facteurs directement impliqués dans la performance sportive, la motivation représente donc un élément clé du mental d'un sportif. Générer et entretenir les attitudes motivationnelles les plus adaptatives à l'égard de la performance constitue un des objets de la préparation mentale. En raison de la permanence de l'action de telles attitudes, cette préparation est à comprendre comme une véritable entreprise éducative destinée à les stabiliser.

\section{2 - LES FACTEURS RESPONSABLES DE LA MOTIVATION DU SPORTIF}

Les modèles théoriques qui ont le plus contribué à la mise au jour des facteurs impliqués dans la motivation d'accomplissement peuvent se regrouper à l'intérieur de deux courants principaux : les modèles centrés sur le rôle des perceptions de contrôle et ceux s'intéressant aux représentations de la compétence. Lorsqu'elle envisage l'application des théories explicatives des comportements de découragement au champ de la pratique sportive, Dweck (1980) souligne en effet que "l'élément clé pour l'individu résigné est ce qui déclenche en lui des sentiments d'incompétence ou d'échec inévitable dî̀ à une perte de contrôle » (p. 9).

2.1 - Les perceptions de contrôle 
La notion de contrôle en psychologie de la motivation revêt actuellement des significations différentes d'une théorie à l'autre. La théorie de la résignation apprise élaborée par Seligman et ses collaborateurs (Seligman, 1975 ; Abramson, Seligman, \& Teasdale, 1978 ; Peterson, Maier, \& Seligman, 1993) considère la notion de contrôle en terme de relation perçue par une personne entre les comportements que celle-ci peut mettre en œuvre et les résultats auxquels elle peut s'attendre. La perception d'une indépendance entre comportement et résultat associée à certaines représentations relatives à la causalité des événements serait à l'origine de comportements résignés et d'affects émotionnels négatifs. La théorie de l'évaluation cognitive de Deci et Ryan $(1980,1985)$ envisage sous le terme contrôle ce qui détermine l'adoption d'un comportement chez un individu. Lorsque ce déterminant a une origine interne, cet individu s'engage dans une activité de manière libre. Lorsque l'origine est externe, les actes répondent à des pressions plus ou moins saillantes issues de l'environnement. Par la dégradation du sentiment d'autodétermination qu'elles provoquent, ces pressions affecteraient l'aspect spontané et libre de l'engagement de l'individu dénommé motivation intrinsèque. Des conceptions différentes du contrôle sont donc sollicitées par les deux modèles théoriques. Pour résumer : la théorie de la résignation apprise envisage la perception du contrôle que l'on peut exercer sur les événements, la théorie de l'évaluation cognitive, quant à elle, s'intéresse à la perception de contrôle de son comportement.

\subsection{1 - Perceptions de contrôle sur la causalité des événements}

L'idée de contrôle de l'environnement et de la nature causale des événements fait depuis longtemps l'objet de nombreuses interrogations. Heider (1958) sous-tend que la recherche de la causalité des événements que l'on peut attribuer à des facteurs personnels ou à des facteurs impersonnels participe à la quête d'un équilibre cognitif entre soi et l'environnement. Dans cette perspective, la notion de contrôle peut donc se rapporter à la perception d'une relation entre son propre comportement et les modifications de cet environnement.

La recherche d'un tel sentiment de contrôle conduit parfois les individus à tenter de se persuader qu'ils sont à l'origine des événements positifs qui leur arrivent. Une telle exagération est désignée sous les termes d'erreur fondamentale (Ross, 1977) ou illusion de contrôle (Langer, 1975). Pour Thill (1987, 1989), certaines pratiques rituelles ou superstitieuses très répandues dans le sport serviraient à alimenter les croyances des athlètes en une forme de contrôle exercé par eux sur une situation perçue comme peu contrôlable. La perception d'une absence de relation entre comportement et résultats réels ou espérés correspond à ce que Seligman et ses collaborateurs dénomment résignation apprise. Il s'agit de "l'apprentissage ou la perception d'indépendance entre la réponse émise par l'organisme et la soumission et/ou la soustraction à un événement désagréable » (Seligman, Maier, \& 
Geer, 1968, p. 258). La résignation apprise peut s'installer à la suite d'une situation incontrôlable contribuant à faire percevoir chez un individu une indépendance entre les comportements qu'il pourra ultérieurement tenter de mettre en œuvre et les résultats qu'il sera susceptible d'obtenir. Ainsi, chaque fois qu'un individu échoue devant une situation dans laquelle il ne perçoit aucun moyen d'atteindre le but, celui-ci fait l'apprentissage de l'inutilité de ses efforts et préfère alors adopter une attitude résignée plus économique. Les conséquences se manifestent alors tant au plan cognitif (échec dans la recherche d'une solution) que dans les registres motivationnel (réduction des efforts, abandon) et émotionnel (anxiété, tristesse, hostilité, colère), pouvant aller jusqu'à affecter l'estime de soi. Il se peut que la résignation s'étende au-delà de la situation même qui en a généré l'apprentissage (Hiroto \& Seligman, 1975). Dans ce cas, la résignation revêt un caractère invasif. Les caractéristiques de la résignation, comme son étendue par exemple, sont déterminées par certaines représentations jouant le rôle de médiateurs cognitifs. L'importance du rôle de ces représentations mérite que l'on s'y attarde quelque peu.

La manifestation d'une attitude résignée acquise à la suite d'une expérience d'incontrôlabilité ainsi que sa généralisation à des situations différentes n'a pas été confirmée dans toutes les études. En fait, l'ampleur de cette manifestation dépend également de certains médiateurs cognitifs : les attributions causales. L'homme cherche à comprendre les causes qui ont pu intervenir sur son environnement. La réponse à la question "pourquoi ?" apparaît alors au travers d'attributions qui sont des représentations construites a posteriori sur la nature causale des événements. En effet, pour les auteurs à l'origine des théories de l'attribution (Heider, 1958 ; Jones \& Davis, 1965 ; Kelley, 1967), les hommes ont tendance à établir des attributions afin de rendre l'environnement prédictible et contrôlable. Ces cognitions élaborées à partir de perceptions de l'environnement influencent en retour nos actions sur le monde. Les processus d'attribution « sont censés renvoyer à la compréhension que l'on se forge de la structure causale du monde, et partant, déterminer pour une large part nos interactions avec ce monde » (Deschamps, 1987, p. 86). Pour Abramson et al. (1978), trois dimensions caractérisant les attributions causales impliquées dans la résignation apprise apparaissent déterminantes dans la construction du sentiment de contrôle et de ses conséquences comportementales. Celles-ci sont relatives au lieu, à la constance temporelle et à la constance structurelle de la causalité.

Le locus de causalité concerne la dimension attributionnelle interne (attributions à des facteurs personnels) versus externe (attributions à des facteurs situationnels) des causes invoquées par les individus. Pour Weiner $(1985,1986)$, il s'agit d'une perception du siège de la cause à ne pas confondre avec son caractère contrôlable, la contrôlabilité représentant alors, pour cet auteur, une dimension attributionnelle supplémentaire distincte du locus. La résignation met en jeu tantôt des attributions à des causes internes, tantôt des attributions à des causes externes. Abramson et al. (1978) parlent dans le premier cas d'une résignation apprise personnelle (" c'est moi seul qui suis incapable de réussir »), préjudiciable pour 
l'estime de soi, et dans le second cas d'une résignation apprise universelle (" personne ne pourrait réussir cette tâche »).

La stabilité désigne la régularité temporelle des causes de succès ou d'échec invoquées. Des attributions d'instabilité associées à un locus de causalité externe (e.g., la malchance) peuvent engendrer de la résignation (Hiroto, 1974). Mais la résignation présente des déficits plus importants lorsque les échecs sont attribués à des causes stables. Weiner $(1985,1986)$ considère, en effet, les attributions à des causes stables comme principalement responsables des états émotionnels de désespoir. Attribuer ses échecs à des causes internes deviendra préjudiciable lorsque ces mêmes causes seront perçues comme étant permanentes (e.g., une faible aptitude). La résignation accompagnée d'attributions à des causes stables est dite chronique tandis qu'elle est qualifiée de transitoire dans le cas opposé (Abramson et al., 1978).

La constance structurelle ou globalité, pour Abramson et al. (1978), réfère au caractère global versus spécifique que les sujets attribuent à la cause perçue comme responsable d'un événement. Tout se passe comme si la personne se pose la question : " la cause responsable de mon échec va-t-elle me faire échouer seulement dans cette situation ou bien également dans d'autres pourtant bien différentes de celle-ci ? "Cette dernière dimension attributionnelle est évidemment directement liée à la généralisation de la résignation (Alloy, Peterson, Abramson, \& Seligman, 1984). Elle est censée déterminer si la résignation va se manifester seulement dans la tâche ou le type de tâche qui a contribué à son apparition ou bien si les déficits vont aussi se prolonger dans des situations différentes. Abramson et al. qualifient alors la résignation de spécifique ou globale.

Si les profils attributionnels relèvent de l'analyse causale portée sur des événements particuliers, les auteurs s'intéressant à la résignation sous-tendent également l'existence de véritables styles attributionnels. Ces styles correspondent, chez les individus, à des tendances relativement permanentes pouvant se manifester dans des circonstances variées. Il est possible de les mesurer à l'aide de l'Attributional Style Questionnaire (ASQ) de Peterson, Semmel, Von Bayer, Abramson, Metalsky et Seligman (1982). En utilisant notamment l'ASQ, Prapavessis et Carron (1988) observent que des joueurs et joueuses de tennis de haut niveau coutumiers des comportements résignés ont tendance à attribuer leurs échecs à des causes internes, stables et globales. Pour Seligman (1990), si le locus de causalité joue un rôle important en ce qui concerne l'estime de soi, la stabilité et la globalité déterminent davantage le style optimiste versus pessimiste des personnes. Ces deux dimensions attributionnelles seraient en lien direct avec l'espérance qu'entretiennent les gens vis-à-vis des événements futurs. Ainsi, après un événement négatif, les individus présentant une tendance aux attributions à des causes instables et spécifiques (e.g., " Aujourd'hui, l'équipe qui nous a battus a parfaitement verrouillé sa charnière centrale en défense ») s'avèrent être les plus optimistes et manifestent les attitudes les plus persévérantes. En revanche, les styles attributionnels présentant les caractères stable et global (e.g., "Que pouvons-nous faire ? La 
différence de points entre les meilleures équipes du championnat et nous est révélatrice de leur supériorité " se retrouvent chez les plus pessimistes et les moins combatifs. De nombreuses interviews de sportifs relevées dans la presse spécialisée américaine montrent que les styles attributionnels de certaines équipes professionnelles de base-ball et de basketball se révèlent être prédicteurs des performances de celles-ci pour la saison en cours et pour la suivante, particulièrement vis-à-vis des matches importants empreints d'une forte pression (Rettew, Reivich, Peterson, Seligman, \& Seligman, 1990 ; Seligman \& Peterson, 1986). Seligman et son équipe ont voulu tester le modèle de l'optimisme chez le nageur international Matt Biondi quatre mois avant les Jeux Olympiques de Séoul en 1988. Biondi avait été évalué à l'aide de l'ASQ comme appartenant à la catégorie des sujets les plus optimistes. Lors d'un entraînement, à la demande des chercheurs, Nort Thornton, le coach du champion, a simulé un événement désagréable en faisant nager à celui-ci un test sur 100 yards papillon. Biondi réalisa le temps respectable de 50.2 secondes mais Thornton lui annonça un temps très moyen de 51.7 secondes, ce qui ne manqua de provoquer chez le nageur une réaction de surprise et de désappointement. Après quelques minutes de repos, Biondi réalisa un second 100 yards dans un temps de 50.0 secondes ! Afin de confirmer l'éventuelle relation entre le style attributionnel et la réaction de Biondi, Seligman, Nolen-Hoeksema, Thornton et Thornton (1990) ont réédité cette expérience avec le reste de l'équipe de nageurs de Berkeley. Ceux d'entre eux qui furent identifiés par l'ASQ comme optimistes ont réalisé une seconde performance supérieure ou égale à la première tandis que les pessimistes ont régressé lors de leur deuxième tentative. Devant l'importance du rôle joué par de telles tendances sur la performance sportive, Hanrahan et ses collaborateurs (Hanrahan \& Grove, 1990 ; Hanrahan, Grove, \& Hattie, 1989) ont créé un questionnaire adapté au sport, le Sport Attributional Style Scale (SASS), qui ajoute d'autres dimensions attributionnelles comme la contrôlabilité (Weiner, 1985, 1986) et l'intentionalité (Elig \& Frieze, 1979) à celles constitutives des styles identifiés par Seligman.

Si la résignation peut s'acquérir après une expérience d'incontrôlabilité des résultats, les déficits cognitifs, motivationnels, émotionnels et en terme d'estime de soi seront d'autant plus profonds que les causes de l'échec apparaîtront internes, stables et globales aux yeux du sportif. Ainsi, Seligman et ses collaborateurs ont montré qu'une résignation associée à ce type de profil attributionnel pouvait être à l'origine de la dépression chez l'être humain (Peterson et al. 1993 ; Peterson \& Seligman, 1984 ; Sweeney, Anderson, \& Bailey, 1986). Par ailleurs, la gravité des perturbations dues à la résignation apprise s'accentue avec l'importance (valence de l'échec et du succès) que revêt la situation aux yeux du sujet (Miller \& Norman, 1979 ; Roth, 1980). En revanche, attribuer un échec à des causes externes, instables et spécifiques relève d'une attitude optimiste permettant d'envisager l'avenir comme dépendant de ses propres efforts. Evidemment, après un succès, les patrons attributionnels décrits ci-dessus inversent les caractéristiques adaptatives ou non adaptatives des attitudes. 
D'une manière générale, on peut donc considérer qu'un état de résignation peut s'installer chez un sportif à la suite d'une situation qu'il n'aura pu contrôler. Mais c'est moins la contrôlabilité objective de cette situation que la manière dont il perçoit puis estime pour l'avenir ses possibilités de contrôle qui sera responsable de l'ampleur des déficits associés à cette résignation. Des attributions causales qui dépendent à la fois d'une analyse ponctuelle et du style attributionnel propre à chaque individu participent à la construction du caractère optimiste ou pessimiste des estimations ainsi élaborées.

\subsection{2 - Perceptions de contrôle de son comportement et autodétermination}

A l'instar de Heider, de Charms (1968) souligne la tendance de l'être humain à vouloir se sentir cause. Toutefois, ce dernier étend la notion de causalité des événements à celle d'origine du comportement. Pour de Charms, les individus préfèrent se percevoir comme étant eux-mêmes la source de contrôle de leurs comportements plutôt que de se sentir contrôlés de l'extérieur comme de simples pions. En mettent l'accent sur l'importance du besoin d'autodétermination chez l'homme, les théories plus récentes de la motivation intrinsèque et de l'évaluation cognitive de Deci et ses collaborateurs s'inscrivent dans la lignée de cette conception élargie du sentiment de contrôle initiée par de Charms.

Le fait qu'une personne s'engage et persiste librement dans une activité pour le seul plaisir que celle-ci lui procure relève d'une motivation intrinsèque. Un cas de motivation intrinsèque particulièrement extrême correspond à ce que Csikszentmihalyi $(1975,1990)$ dénomme flow. Le flow traduit un état d'absorption et d'implication totales dans une tâche, à tel point que le contrôle de ses actes échappe à la conscience de l'individu. Csikszentmihalyi a observé ce phénomène chez des personnes pratiquant intensément et gratuitement certaines activités comme la musique, le jeu d'échecs mais aussi des activités physiques comme l'escalade, la danse, l'athlétisme amateur. Un tel état se rencontre le plus souvent lorsque la tâche représente pour un individu un niveau de challenge élevé mais que celui-ci possède également un haut niveau d'habileté. En sport, Jackson (1995) a remarqué que l'accès à un état de flow était notamment influencé par la qualité de la préparation (physique et mentale), la confiance, la concentration, les sensations concernant l'évolution de ses performances, l'envie de réussir ses challenges ainsi que par un état d'activation optimum, spécifique à chaque athlète.

D'un autre côté, les comportements extrinsèquement motivés répondent à des incitations externes surajoutées au motif intrinsèque d'une tâche. Deci (1975) met en avant le fait qu'une motivation extrinsèque présente, dans la plupart des cas, l'inconvénient de détourner la motivation du sujet pour réaliser une tâche au profit d'un but à atteindre plus saillant (e.g., obtenir une récompense, une gratification). Un phénomène de surjustification se produit alors (Lepper, Greene, \& Nisbett, 1973). Il en résulte un abandon fréquent de l'activité dès lors que ce dernier but est atteint ou supprimé. On peut donc succinctement 
résumer le problème posé par la motivation extrinsèque en retenant que si certaines pratiques ont le pouvoir de susciter l'engagement d'un individu dans une tâche, celles-ci reviennent à effectuer une substitution de but. Ceci contribue, malgré la poursuite de l'activité par le sujet, à réduire son investissement sur la tâche initiale proprement dite. En outre, si ce type de motivation ne semble pas affecter l'activité apparente du sujet, à long terme, un effet négatif s'observe fréquemment sous la forme d'une réduction de l'activité voire d'un abandon. La plupart de ces études ont porté sur des tâches essentiellement cognitives comme la réalisation de puzzles. Cependant, des effets similaires concernant l'érosion de la persévérance ont été observés par Orlick et Mosher (1978) dans une étude portant sur des tâches motrices. Ces auteurs ont remarqué que la persévérance dans une épreuve d'équilibre sur stabilomètre diminuait chez des enfants de dix ans qui avaient été récompensés pour leur participation tandis qu'elle augmentait chez ceux qui n'avaient pas été récompensés.

Malgré sa validité expérimentale, la généralisation de l'hypothèse de surjustification requiert une certaine prudence (Deci \& Ryan, 1985 ; Weiss \& Chaumeton, 1992). Une récompense externe n'entraîne pas toujours une diminution de la motivation intrinsèque. Son effet dépend notamment de l'intérêt que porte le sujet à la tâche. Ainsi, Calder et Staw (1975) ont montré à partir de tâches de puzzle que les récompenses affectaient négativement la motivation intrinsèque des individus percevant l'activité comme intéressante tandis qu'elles augmentaient la motivation intrinsèque pour ceux qui percevaient initialement l'activité comme inintéressante. Dans les activités sportives, l'ensemble des tâches à réaliser ne présentent pas toutes un intérêt équivalent. L'usage de certaines gratifications pourrait donc parfois s'avérer utile, particulièrement lors de phases d'entraînement peu attrayantes. Par ailleurs, de nombreuses recherches en laboratoire ont utilisé des récompenses sans rapport avec les performances des participants. Il s'avère pourtant que les feed-back donnés par des professeurs ou des entraîneurs en relation avec les résultats (Horn, 1987) ou bien les récompenses offertes en fonction des performances obtenues dans une tâche de lancer (Thomas \& Tennant, 1978) augmentent la motivation intrinsèque. Enfin, les effets des récompenses dépendent également des personnes qui les reçoivent, leurs caractéristiques individuelles influençant la signification fonctionnelle accordée à la gratification. Ryan (1980) a montré, par exemple, que des footballeurs américains étaient de moins en moins motivés intrinsèquement au fil des années pendant lesquelles ils percevaient une rémunération. De plus, la motivation intrinsèque de ces mêmes footballeurs était plus faible que celle de footballeurs non payés. En revanche, des lutteuses rétribuées étaient davantage intrinsèquement motivées que leurs partenaires non payées. Pour Ryan, cette divergence de résultats proviendrait de la différence qui existerait entre les significations fonctionnelles accordées au salaire par les sujets vis-à-vis de leurs activités sportives respectives. Pour les footballeurs, le salaire aurait été compris comme un moyen de contrôle voire de pression, celui-ci pouvant être retiré en cas de contre-performance. Pour les lutteuses, la rémunération aurait véhiculé l'information d'une compétence particulière dans la mesure ou toutes les 
sportives ne percevaient pas d'argent. Toujours dans le domaine sportif, Thill et Mouanda ont aussi relativisé les effets négatifs de certaines récompenses sur la motivation intrinsèque. Ces auteurs (Mouanda, 1989 ; Thill \& Mouanda, 1990) mettent en avant le rôle du contexte d'allocation de la récompense. Les rétributions monétaires attribuées individuellement à des handballeurs à l'issue de tâches de tirs au but ont entrainé chez eux une diminution de la motivation intrinsèque moins importante que des attributions collectives. Là encore, l'attribution individuelle d'une récompense fournirait au joueur une information de compétence tandis qu'une allocation collective revêtirait un aspect plus contrôlant. Pour Deci et Ryan (1980, 1985), la présence de renforçateurs externes n'affecterait pas la motivation intrinsèque tant que ceux-ci véhiculeraient une information de contrôle ou de compétence. La signification fonctionnelle accordée par un individu à ces renforçateurs constituerait le facteur déterminant du sentiment d'autodétermination et de la motivation intrinsèque.

Aujourd'hui, la dichotomie motivation intrinsèque - motivation extrinsèque fait place à une conception plus graduelle de la notion d'autodétermination. Pour Deci et Ryan (1985), il existerait une sorte de continuum dont les différents degrés correspondraient à des formes différentes de la motivation extrinsèque. A partir de ce principe, Brière, Vallerand, Blais et Pelletier (1995) ont conçu un questionnaire, l'Échelle de Motivation dans les Sports (ÉMS), destiné à mesurer le niveau d'adhésion des individus aux différentes formes de motifs dans le domaine sportif ainsi que l'absence de motivation à l'égard du sport. En suivant une voie de plus en plus instrumentalisée, le continnum d'autodétermination est hiérarchisé de la manière suivante (certains exemples utilisés pour illustrer les concepts de Deci et Ryan sont adaptés à partir de l'ÉMS de Brière et ses collaborateurs) :

- La régulation intégrée correspond à la forme de motivation extrinsèque la plus autodéterminée. Les actions sont mises en œuvre parce qu'elles s'inscrivent dans des projets congruents vis-à-vis de la personnalité d'un individu. C'est le cas lorsqu'une personne dynamique et active fait du sport parce que le sport véhicule et développe ces qualités.

- Au niveau de l'identification, les actions répondent à un double investissement : pour elles-mêmes et pour les bénéfices qui leur sont associés. Ainsi, on peut faire du sport parce que c'est aussi l'occasion d'apprendre des choses qui peuvent être utiles dans la vie.

- La régulation introjectée correspond à des actions contrôlées par des règles (pressions, contrôles) imposées par soi-même. Par exemple, faire du sport parce que c'est absolument nécessaire si l'on veut rester en forme.

- La régulation externe concerne les actions les plus instrumentales. Elles sont contrôlées par des perspectives de gratification, de récompense ou bien par la menace d'événements désagréables (sanctions). C'est le cas lorsqu'on pratique un sport pour le prestige d'être un athlète ou pour être bien vu par les autres.

- L'amotivation, en marge de ce continuum, représente l'absence totale de motivation.

En s'intéressant aux différents niveaux d'instrumentalisation de l'activité d'un athlète vis-à-vis des buts que celui-ci peut poursuivre, le continuum d'autodétermination permet de 
comprendre ce qui l'incite à accepter les nombeuses contraintes inhérentes à la pratique d'un sport au plus haut niveau. Entre les deux extrêmes que représentent une motivation intrinsèque et la régulation externe, il existe donc des paliers intermédiaires dont certains garantissent une autodétermination relative (régulations intégrée et identifiée notamment).

Finalement, il apparaît que les actions les plus intrinsèquement motivées telles qu'elles sont caractérisées lors des états de flow, par exemple, sont à rechercher en raison de leurs effets motivationnels. Il faut bien reconnaître, cependant que le sportif peut difficilement échapper à certaines incitations externes comme l'argent ou le prestige. Dans ce cas, l'aspect contrôlant de ces agents externes ne doit pas devenir plus saillant que les sentiments d'autodétermination et de compétence qui correspondent aux représentations les plus à même de générer des comportements persévérants.

\section{2 - Le sentiment de compétence}

La notion de sentiment de compétence peut revêtir différentes significations selon l'étendue du champ dans lequel ce sentiment est censé s'exercer. Dans son acceptation la plus restreinte, le sentiment de compétence peut être comparé à la confiance dans le mouvement (Griffin \& Keogh, 1981, 1982) que le sujet ressent avant l'exécution d'un geste. Lorsque la confiance se réfère à une tâche particulière, le sentiment de compétence est à rapprocher d'une expectation d'efficacité personnelle (Bandura, 1977, 1997) que l'on peut définir comme la probabilité subjective de posséder les capacités nécessaires pour atteindre un résultat. Si on la considère comme finalisée par la réalisation efficace et économique d'une performance motrice, cette capacité renvoie à la notion d'habileté motrice (Guthrie, 1957). On peut alors exprimer le sentiment de compétence en termes d'habileté perçue. Mais celui-ci peut revêtir un caractère plus général et recouvrir une même classe d'activités comme les activités physiques (Harter, 1982, 1990). Pour cet auteur, la compétence perçue à l'égard des activités sportives contribue, avec la compétence scolaire, les relations sociales, l'apparence physique et le comportement social, à la construction d'une estime de soi entendue comme la valeur générale qu'un individu attribue à sa personne.

Pour White (1959), les comportements d'accomplissement seraient mus par un besoin de se sentir compétent. Le fait que le sentiment de compétence puisse être considéré comme un facteur important de la motivation d'accomplissement a suscité de nombreuses recherches initiées essentiellement dans le domaine scolaire (Dweck, 1986 ; Nicholls, 1984a, 1984b) et poursuivies dans celui des activités physiques et sportives (voir Duda, 1992, 1993 ; Roberts, 1992 ; Weiss \& Chaumeton, 1992, pour une revue).

\subsection{1 - Représentations de la compétence et buts d'accomplissement}


Selon Nicholls, il existe deux manières différentes utilisées par les individus pour se représenter leur compétence. La première, auto-référencée, met en jeu une comparaison se référant à des standards internes ou bien objectifs ; l'attention du sujet se porte alors sur ses progrès personnels ou sur la réussite d'une tâche particulière. La seconde, normativement référencée, sollicite un processus de comparaison sociale, les résultats ne revêtant un caractère incitatif que dans la mesure ou ceux-ci permettent la mise en évidence d'une habileté supérieure à celle des autres. Selon la manière adoptée pour se représenter leur compétence, les personnes auront tendance à se fixer deux types de buts. Dans le cas d'une représentation auto-référencée, elles s'orienteront préférentiellement vers des buts investis sur la maîtrise des tâches (Nicholls, 1984a ; 1984b) encore appelés buts d'apprentissage (Dweck, 1986) ou buts de maîtrise (Ames, 1984). Une représentation normativement référencée incitera à l'adoption de buts d'implication de l'ego (Nicholls, 1984a ; 1984b) également dénommés buts de performance (Dweck, 1986) ou buts centrés sur l'aptitude (Ames, 1984).

Pour ces auteurs, le type de but développé combiné avec le niveau d'habileté perçue entraîne d'importantes conséquences motivationnelles. Une personne s'estimant habile ou compétente dans un domaine particulier manifestera des comportements adaptatifs, ceci quel que soit le type de but développé (investi sur la tâche ou sur l'ego). En revanche, si elle s'estime incompétente, cette personne s'attachera à déployer des efforts seulement si elle poursuit des buts investis sur la tâche. Si par contre ses buts motivationnels sont orientés vers l'ego, elle mettra en œuvre des stratégies visant à échapper aux jugements défavorables des autres en choisissant des tâches présentant un niveau de difficulté faible (assurant le succès) ou au contraire très élevé (dans lesquelles un échec n'est pas déshonorant). Lorsque l'évitement d'une tâche de difficulté optimale par rapport à ses propres ressources sera impossible, cette personne cherchera à protéger son ego en montrant de manière évidente qu'elle échoue parce qu'elle ne fait aucun effort. Ainsi, aux yeux des autres, sa performance ne pourra être attribuée à une insuffisance d'habileté.

Dans le domaine sportif, Burton (1989) a testé chez des nageurs de compétition les effets d'un programme d'entraînement à la fixation de buts de maîtrise (que l'auteur dénomme buts de performance en opposition aux buts de résultat) portant sur cinq mois. Ce programme comprenait :

- des informations sur l'intérêt des buts de maîtrise

- la lecture d'un manuel d'entraînement à la fixation de buts

- la tenue d'un journal d'entraînement (avec relevé des buts)

- cinq sessions collectives d'apprentissage et d'entraînement des habiletés à se fixer des buts de maîtrise

- cinq sessions individuelles d'apprentissage et d'entraînement des habiletés à se fixer des buts de maîtrise (informations sur l'intérêt de ce type de buts, mise au jour du journal, exercices du manuel, techniques d'imagerie de base et spécifique aux buts de maitrise. 
Les nageurs qui se sont avérés les plus habiles à adopter ce type de buts à l'issue du programme ont manifesté les sentiments de compétence ainsi que les progrès de performance les plus élevés. Si l'efficacité des buts de maîtrise se confirme donc en sport, on ne peut cependant pas se prononcer sur le caractère préjudiciable des buts de promotion de l'ego dans ce contexte dans la mesure ou l'étude de Burton n'a pas envisagé la comparaison des deux types de but. Sur ce plan, Locke et Latham (1985) affirment que la compétition, par la comparaison sociale qu'elle instaure, est censée améliorer la performance en incitant le sportif à se fixer des objectifs plus élevés et en augmentant son engagement vers ces objectifs. Dans une récente méta-analyse reprenant les données issues de 36 études publiées sur la fixation de buts dans les activités physiques ou sportives, Kyllo et Landers (1995) relèvent que les buts centrés sur les résultats compétitifs engendrent des performances plus élevées que les buts auto-référencés. Quoiqu'il en soit, la poursuite de buts de compétition, difficilement dissociable de la pratique sportive, ne semble pas forcément préjudiciable à la motivation du sportif. Il importe alors de veiller à ce que celle-ci ait pour corollaire l'adoption de buts de maîtrise ce qui est envisageable dans la mesure où les deux types de buts ne semblent pas incompatibles. Si pour Dweck (1986), ces buts constituent les deux pôles extrêmes d'une même dimension, pour Nicholls (1984a, 1989, 1992), il s'agit plutôt de deux dimensions indépendantes et donc non antinomiques. Le caractère indépendant de ces buts a été confirmé en sport par Roberts, Treasure et Kavussanu (1996). Aussi, la compétition peut très bien générer des buts de maîtrise (Roberts, 1992) et l'absence de compétition peut même s'avérer préjudiciable à la poursuite de buts de maîtrise. Nous l'avons constaté (Gernigon \& Le Bars, 1995) en comparant les effets de la pratique de deux sports de combat, l'un anti-compétitif, l'aïkido, l'autre compétitif, le judo, sur les buts motivationnels adoptés par leurs pratiquants respectifs. Nous avons remarqué que les pratiquants d'aïkido de niveau ceinture noire présentaient des orientations conjointement moins marquées vers l'ego et moins marquées vers la tâche que les débutants. Par contre, les "ceinture noire" de judo se déterminaient davantage vers l'ego que les débutants tout en maintenant leur niveau d'engagement vers la maîtrise identique à ces derniers. Nous avons donc émis l'hypothèse que l'absence de compétition, caractéristique de l'aïkido, pourrait créer une carence d'objectifs, lesquels auraient été susceptibles de nourrir de nouveaux buts de maîtrise et de progrès. En revanche, l'adhésion croissante aux buts de promotion de l'ego observée au fil des années de pratique d'un sport compétitif comme le judo n'affecterait pas la poursuite de buts de maîtrise. Ces résultats confirment le point de vue de Roberts et al. (1996), pour qui il serait inutile de chercher à supprimer les buts de promotion de l'ego pour les remplacer par des buts d'investissement sur la tâche, les athlètes à risque au plan motivationnel étant seulement ceux qui sont à la fois fortement orientés vers l'ego et faiblement orientés vers la tâche. En fait, comme le suggère Duda (1988), les sportifs investis à la fois sur l'ego et sur la tâche bénéficient de deux sources de succès et donc de plusieurs raisons d'entretenir leur motivation. 
L'orientation des buts vers la maîtrise de la tâche ou vers la promotion de l'ego influence également certaines variables émotionnelles comme l'anxiété (Nicholls, 1989). Pour cet auteur, une orientation vers l'ego entraînerait un état d'anxiété qui serait responsable des préjudices observés sur la performance. Avant une compétition sportive importante, l'incertitude du résultat associée aux enjeux que revêt un succès ou un échec est également source d'anxiété (Martens, 1977 ; Martens, Vealey, \& Burton, 1990). Un investissement dirigé quasi exclusivement vers la comparaison sociale risquerait alors d'exacerber l'état d'anxiété généré par la situation de compétition. Duda, Newton et Chi (1990) ont effectivement montré qu'avant une compétition, des joueurs de tennis très orientés vers l'ego et peu optimistes quant à l'issue du match présentaient une confiance en soi faible et un état d'anxiété élevé. Par ailleurs, Hall et Kerr (1997) ont constaté qu'avant une compétition, de jeunes escrimeurs étaient d'autant plus anxieux qu'ils étaient fortement orientés vers l'ego et faiblement investis sur la tâche.

\subsection{2 - Détermination des orientations motivationnelles}

L'importance du rôle des buts d'accomplissement dans l'adoption de patrons adaptatifs ou mal adaptatifs vis-à-vis de la performance invite à s'interroger sur la genèse de ceux-ci. Plusieurs types de facteurs semblent participer à la détermination des orientations motivationnelles : il s'agit de facteurs développementaux, différentiels, culturels et contextuels.

\section{Facteurs développementaux}

La représentation qu'un enfant peut se construire à propos de son habileté se précise vers 11-12 ans. Celle-ci devient plus objective et se différencie de la notion d'effort (Nicholls, 1978 ; Nicholls \& Miller, 1984). Les enfants deviennent alors capables d'identifier les causes de leurs succès ou de leurs échecs et donc de les attribuer soit à leur niveau d'habileté, soit à leurs efforts. Ainsi, pour Roberts (1984), c'est à partir de cet âge (quand la possibilité de se représenter objectivement sa compétence semble acquise) que les enfants développant des buts d'investissement de l'ego fuient les sports de compétition lorsqu'ils s'y perçoivent peu habiles.

\section{Facteurs différentiels}

Les orientations motivationnelles répondent à des caractéristiques relativement stables de la personnalité, certains individus poursuivant de manière régulière un type de but plus qu'un autre. Les différents contextes auxquels une personne est confrontée au cours de son développement contribuent à la genèse de ces traits stables. De plus, des différences entre garçons et filles sont observables. Les garçons semblent plus investis sur l'ego tandis que les filles s'orientent davantage vers la maîtrise des tâches (Duda, 1988). De même, les garçons 
manifestent un esprit de compétition plus exacerbé que les filles, ces dernières étant plus intéressées par l'atteinte des objectifs fixés (Gill, 1986,1988). Nous avons observé chez de jeunes adultes une imperméabilité des garçons aux consignes et aux contraintes de coopération formulées vis-à-vis d'une tâche d'apprentissage collectif (en binôme) d'une habileté aquatique (Gernigon, d'Arripe-Longueville, \& Huet, 1996). Alors que ces garçons ont poursuivi conjointement des buts auto-référencés et des buts normativement référencés (de promotion de l'ego et même d'hyper compétitivité) malgré les consignes, les filles se sont préférentiellement référées à des standards de comparaison internes.

\section{Facteurs culturels}

Les études transculturelles concernant les orientations motivationnelles en termes de buts sont peu nombreuses. Pourtant, il semble bien que des différences soient observables entre les athlètes aux origines ethniques différentes. L'intervention auprès du sportif devrait pouvoir tenir compte de ces différences. C'est pourquoi, pour Duda et Allison (1990), les études inter-ethniques relatives aux buts motivationnels devraient être plus largement développées. Pour l'heure, il ressort que les anglo-saxons sont davantage orientés vers la comparaison sociale, vers la démonstration de la compétence et vers la compétition que les individus d'origine espagnole (Duda, 1985, 1986a), noire (Duda, 1986a) et les Navajos (Duda, 1986b). Devant ces résultats qui n'intéressent que partiellement l'entraîneur sportif français, on ne peut que regretter l'absence de données comparatives avec des sportifs européens.

\section{Facteurs contextuels}

Dans le milieu familial, les orientations motivationnelles des parents agissent sur la signification accordée au succès sportif (Roberts, Treasure, \& Hall, 1994). Ces auteurs remarquent que les parents orientés vers l'ego s'intéressent surtout au nombre de victoires de leur enfant ou bien encore aux performances de celui-ci comparées à celles de ses camarades. En revanche, lorsqu'une telle orientation est moins marquée, les parents s'intéressent davantage à l'intégration de leur enfant au sein d'une équipe, les victoires en compétition étant alors considérées comme secondaires. L'interprétation que font les parents de la réussite sportive tend à inciter leurs enfants à poursuivre des buts de promotion de l'ego ou au contraire des objectifs de maîtrise de la tâche. Bien que le climat motivationnel existant dans la cellule familiale semble peu maléable, Weinberg et Gould (1995) suggèrent d'associer l'entourage du jeune à sa vie sportive ainsi qu'à la vie du club afin de sensibiliser sa famille, ses amis sur l'importance des buts de maîtrise et des progrès personnels à côté des objectifs compétitifs.

Le contexte de pratique influence également ces orientations. S'appuyant sur les résultats de nombreux travaux effectués dans le domaine scolaire (Ames, 1984 ; Ames \& Ames, 1984 ; Ames \& Archer, 1988), les recherches s'intéressant au rôle du climat 
motivationnel dans les activités sportives connaissent un récent développement. Ainsi, White et Duda (1994) ont comparé chez des jeunes et chez des adultes des modalités de pratique sportive marquées par différents niveaux d'engagement vis-à-vis de la compétition. Ces auteurs ont alors remarqué que les contextes mettant le plus l'accent sur la compétition et sur l'habileté normative favorisaient davantage les orientations vers l'ego. Dans notre étude comparative entre un sport de combat non compétitif, l'aïkido, et un sport de combat compétitif, le judo (Gernigon \& Le Bars, 1995), nous avons montré que si les niveaux d'investissement de l'ego d'enfants de 12 ans débutant dans chacune de ces deux activités ne différaient pas, en revanche, ceux des pratiquants confirmés se distinguaient radicalement, les aïkidokas s'éloignant davantage de l'implication de l'ego, tandis que les judokas s'en rapprochaient. Des tendances similaires ont également été observées chez les adultes. Si le choix de pratiquer l'aïkido ou le judo ne semble donc pas avoir été déterminé par une quelconque orientation de départ, ce sont bien les climats de pratique de ces activités qui auraient influencé de manière différenciée les buts d'investissement de l'ego des pratiquants. Par ailleurs, chez des adolescents, Kavussanu et Roberts (1996) ont montré que les perceptions d'un climat orienté vers la tâche, lors d'entraînements en tennis, étaient positivement corrélées au plaisir, aux efforts consentis, à la compétence perçue et au sentiment d'être efficace, tandis que ces mêmes perceptions étaient négativement reliées aux tensions et à la pression ressenties. Au plan émotionnel, Walling, Duda et Chi (1993) ont observé, chez de jeunes sportifs de 14 ans, que la perception d'un climat orienté vers la tâche était positivement corrélée avec la satisfaction d'appartenir à une équipe et négativement corrélée avec l'inquiétude liée au résultat. Par contre, la perception d'un climat orienté vers l'ego était concomitante des phénomènes inverses. Récemment, Theeboom, De Knop et Weiss (1995) ont comparé les effets des deux types de climat chez des enfants de 8 à 12 ans participant à une session de formation en wushu portant sur trois semaines. Les enfants confrontés à un climat de maîtrise ont témoigné davantage de plaisir, un sentiment de compétence et une motivation intrinsèque plus élevés que ceux ayant suivi la session dans un climat de compétition. Ils ont également manifesté de plus grands progrès que ces derniers dans l'acquisition d'une habileté motrice spécifique au wushu.

Pour Roberts et Treasure (1994), il est possible de faire infléchir les dispositions motivationnelles, issues du milieu familial par exemple, en jouant sur la structure du contexte de pratique. Ceci permet d'ouvrir d'éventuelles possibilités d'action pédagogique destinées à viser une éducation d'attitudes propices à la qualité de la préparation à la performance sportive.

En somme, la nature des buts motivationnels poursuivis par les sportifs semble entraîner d'importantes conséquences motivationnelles. L'orientation de ces buts vers la promotion de l'ego ou vers la maîtrise des tâches résulte de nombreux facteurs dont les principaux sont liés à l'âge, aux caratéristiques individuelles, à l'origine culturelle des 
personnes et enfin, au contexte dans lequel ces personnes agissent. En raison de leur caractère non maléable, les trois premiers facteurs apparaissent peu perméables à d'éventuelles tentatives d'intervention de la part de l'entraîneur sur les buts. En revanche, l'action de celui-ci peut s'exercer pleinement sur le contexte de pratique dont il a la responsabilité.

\section{3 - PERSPECTIVES D'APPLICATION}

Qu'elle demeure du ressort de l'entraîneur ou qu'elle émane de l'assistance d'un "préparateur mental", l'intervention auprès du sportif doit se préoccuper du développement et de l'entretien de la motivation de celui-ci. Elle ne peut alors s'affranchir d'une prise en compte des processus psychologiques impliqués dans la motivation d'accomplissement que sont les perceptions de contrôle et de compétence évoquées plus haut. La perspective motivationnelle s'inscrivant forcément dans la durée, les recommandations qui suivent ne se veulent utiles que dans le cadre d'une éducation des attitudes. "Une attitude est un état d'esprit ou une prédisposition générale psychologique envers quelque chose ; cette prédisposition oriente dans un certain sens toutes les interactions avec l'objet en question " (Mucchielli, 1994, p. 13). Ici, les prédispositions à développer ont pour objet la pratique sportive à l'égard de laquelle l'athlète est susceptible de générer certaines représentations de son propre contrôle et de sa compétence. Loin de vouloir constituer un référentiel exhaustif des secteurs d'intervention possibles, ces propositions s'adressent à quelques moments clés de la vie sportive que sont la détermination des objectifs, l'analyse de la prestation passée et, d'une manière générale, le climat motivationnel qui entoure la vie sportive, particulièrement lors de l'entraînement.

\section{1 - La détermination des objectifs}

L'effet des techniques de fixation de buts sur la performance a d'abord été étudié dans le contexte de la psychologie industrielle et organisationnelle. Pour Locke \& Latham (1985), les hypothèses habituellement validées dans ce domaine doivent se vérifier dans celui de la performance sportive. Ainsi, la situation de compétition est censée augmenter les performances. Des buts spécifiques et difficiles doivent s'avérer plus efficaces que des buts vagues et faciles. Des buts à court terme doivent faciliter l'atteinte de buts à plus long terme. L'efficacité des buts s'accroît lorsque ceux-ci sont partagés par l'athlète et lorsque des informations relatives à ses progrès lui sont fournies. Ces caractéristiques des buts influenceraient certains déterminants motivationnels de la performance en jouant sur la quantité d'effort consentie, la persévérance, la volonté de rechercher des stratégies efficaces ainsi que sur l'orientation de l'attention. Toutefois, les tentatives d'application au domaine de la performance sportive ayant vu le jour durant cette décennie mettent en avant des résultats équivoques. L'inconsistance des résultats est autant imputable à des problèmes 
méthodologiques inhérents aux protocoles expérimentaux utilisés dans les recherches (Locke, 1991) qu'à leur manque de pertinence vis-à-vis des activités sportives (Weinberg, 1992 ; Weinberg \& Weigand, 1993, 1996). A partir de leur méta-analyse relative aux nombreuses études publiées sur la fixation de buts dans les activités physiques ou sportives, Kyllo et Landers (1995) concluent que ce ne seraient pas les buts élevés mais les buts de difficulté moyenne qui amélioreraient le plus les performances. De même, il apparaît que si l'intérêt des buts spécifiques serait vérifié, ceux-ci ne s'avéreraient pas plus efficaces que des buts vagues. En revanche, la compétition, l'association de buts à court terme et de buts à long terme ainsi que leur acceptation par le sujet conduiraient bien aux plus grands progrès de performance.

Quoiqu'il en soit, de nombreux entraîneurs sportifs ainsi que les spécialistes en préparation mentale participent à l'élaboration des programmes d'objectifs en s'inspirant des modèles cognitivo-comportementaux (Gernigon, 1998). Les quelques repères qui suivent, adaptés d'après Weinberg et Gould (1995), peuvent servir de guide à la détermination des objectifs sportifs :

1) Fixer des buts spécifiques en des termes précis, si possible chiffrés, ce qui autorisera une évaluation mesurée de leur état d'achèvement.

2) Fixer des buts difficiles mais réalistes. Pour Harter, la plus grande satisfaction s'obtient à partir de succès obtenus dans des tâches suffisamment difficiles. La tâche la plus plaisante et la plus motivante pour un individu est celle qui représente à ses yeux un niveau optimal de défi (Harter, 1978). Il appartient donc à l'entraîneur de trouver avec l'athlète, le juste équilibre entre la difficulté d'un projet et la faisabilité de celui-ci.

3) Fixer des buts à long et à court terme. Il importe, en effet, de baliser les étapes qui mènent aux objectifs les plus distants en temps et en difficulté par des buts plus rapidement accessibles, donc plus motivants. L'atteinte de chaque nouveau sous-but de la hiérarchie qui mène à l'objectif ultime peut être matérialisée sur un graphique. Ceci permet notamment au sportif de visualiser le lien qui unit les différentes étapes mais aussi de prendre conscience de sa progression vers le but le plus éloigné.

4) Fixer des buts de maîtrise. Si les buts de compétition sont bien évidemment inévitables en sport, une focalisation trop grande voire exclusive sur ceux-ci génère une anxiété préjudiciable à la performance. En fait, à chaque but de résultat devraient correspondre plusieurs buts de maîtrise (ou de performance auto-référencés) censés conduire à ce résultat.

5) Ecrire ses propres buts. Ceci présente l'avantage de présenter les objectifs sous la forme de contrats que le sportif peut consulter régulièrement, soit sur fiches, soit dans un cahier ou bien encore sur un poster.

6) Mettre en place des stratégies d'atteinte des buts. Il s'agit de choisir les moyens en termes de procédures à utiliser lors des entraînements pour atteindre les objectifs. Les stratégies adoptées déterminent les tâches à accomplir dans leurs aspects qualitatifs et 
quantitatifs. Une certaine flexibilité des stratégies est souhaitable afin de proposer des manières différentes de parvenir au même but.

7) Prendre en considération la personnalité du sportif. Si les challenges sont bien acceptés par les personnes orientées vers la maîtrise des tâches, il n'en va pas de même pour les sujets trop ou exclusivement orientés vers la comparaison sociale. Ces derniers chercheront plutôt à fuir les défis au profit de buts très faciles à atteindre ou au contraire présentant une difficulté extrême. Pour ces sportifs, il convient donc, avant toute chose, de souligner l'intérêt des buts auto-référencés et de mettre en valeur l'atteinte de ce type de buts ainsi que les progrès personnels, ceci d'autant plus que le sujet est jeune et plus facilement "éducable".

8) Favoriser l'engagement personnel du sportif vers les buts visés. Encourager les progrès, fournir à l'athlète des repères relatifs à ses réalisations, lui laisser une part de décision dans la détermination de ses propres buts.

9) Soutenir le sportif dans ses efforts pour atteindre ses buts. Communiquer avec l'entourage du sportif pour lui signifier l'importance des performances de maitrise à côté de celle habituellement accordée aux résultats.

10) Evaluer les progrès dans la réalisation des buts. Ceci nécessite que les stratégies d'évaluation soient initialement prévues avec le programme de fixation de buts.

\section{2 - L'analyse de la prestation passée}

L'épreuve sportive, en tant qu'instant de vérité, possède une valeur informationnelle entraînant des conséquences sur la motivation et les émotions. L'analyse des résultats et de la manière dont ceux-ci sont obtenus apparaît alors déterminante pour le maintien d'un haut niveau d'engagement du sportif. C'est pourquoi la logique qui anime l'analyse de la prestation passée s'inscrit dans une double dimensionnalité temporelle de récupération et de préparation mentales (Gernigon, 1996). Le résultat passé y apparaît seulement comme l'un des facteurs pouvant influencer les processus cognitifs impliqués dans les comportements futurs d'abandon ou de persévérance. Ces processus ou médiateurs cognitifs sont des représentations relatives au contrôle de la situation par l'athlète et à sa propre compétence.

\subsection{1 - Préserver le sentiment de contrôle des événements}

Le sportif n'échappe pas à une tendance générale qui conduit l'être humain à s'interroger sur le pourquoi de ses résultats. Toutefois, l'impact émotionnel consécutif d'une prestation sportive dégrade l'objectivité de l'analyse causale. Aussi est-il préférable d'éviter toute analyse technique ou tactique "à chaud" d'une prestation sportive.

De l'analyse causale des résultats dépendent des représentations sur la contrôlabilité de l'environnement et des événements. Ces représentations sont à l'origine de conséquences 
déterminantes pour le sportif. Comme le soulignent les travaux de Seligman et de ses collaborateurs, la perception d'indépendance entre les comportements et les résultats obtenus est génératrice de résignation. Les déficits qui en découlent affectent directement la performance, la motivation ainsi que la sphère émotionnelle. Les caractéristiques des causes perçues comme responsables des résultats obtenus jouent sur la gravité des déficits ainsi observés.

Si la mise en avant de causes internes (facteurs personnels) et stables (ces facteurs interviennent régulièrement) après un succès s'avère propice à l'adoption de comportements persévérants, l'attribution d'un échec à ces mêmes causes devient préjudiciable. En revanche, une attribution à des causes internes mais instables, invoquant la quantité d'effort déployée par exemple, préserve la perception de contrôle sur les événements, ceci quel que soit le résultat. Ainsi, l'aspect circonstanciel d'un échec, qui sera perçu comme surmontable pour peu que l'on décide d'investir davantage d'efforts, n'affectera pas la motivation du sujet.

Identifier les causes objectives d'un résultat implique dans tous les cas de souligner le caractère réversible de celles-ci. Les facteurs considérés comme responsables d'un succès devront être perçus comme éphémères en cas de réduction de l'intensité du travail à l'entraînement. Il en va de même pour les raisons d'une contre-performance qui devront apparaître comme labiles si le sportif consent à investir suffisamment d'efforts.

\subsection{2 - Préserver l'estime de soi}

Les perceptions de compétence participent à la construction de l'estime de soi (Harter, 1982, 1990). Le problème réside alors dans la difficulté qu'il y a à préserver ce sentiment de compétence et l'estime de soi après une défaite ou une contre-performance. En fait, le résultat n'aurait pas de valeur incitatrice ou inhibitrice en lui-même si ce n'est dans l'information de compétence ou d'incompétence qu'il fournit au sujet (Deci \& Ryan, 1980, 1985). La construction du sentiment de compétence ne pouvant se faire à partir de succès trop aisés à obtenir, on mesure aisément les effets dévastateurs d'une défaite enregistrée face à un adversaire de plus faible niveau. C'est pourquoi, il importe de ne jamais dévaloriser la tâche ou l'adversaire, ceci quel que soit le résultat. Le contraire présenterait, en cas d'échec, le risque d'affecter gravement l'estime de soi par l'attribution du résultat à une faible compétence.

Le souci de préserver le sentiment de compétence nécessite une organisation temporelle en "sandwich" (positif - négatif - positif) du "debriefing" qui fait suite à une compétition (Thill, 1989). Pour cet auteur, il est préférable d'aborder en premier lieu les points positifs même si ceux-ci sont peu nombreux, notamment en cas de contre-performance. Il est bien rare, en effet, qu'aucun élément de satisfaction ne puisse être enregistré dans un des registres impliqués dans la performance (technique, tactique, énergétique, mental, émotionnel ou relationnel). Dans un second temps, celui de l'analyse causale des aspects négatifs, il 
s'agira davantage de mettre en évidence les facteurs circonstanciels de ses propres faiblesses et de ses points forts ainsi que ceux de l'adversaire avec le maximum d'objectivité et de sérénité. Pour illustrer l'importance de cette analyse critique après un succès, voici les propos de Bob Dwyer, entraîneur de l'équipe Australienne de Rugby, tels qu'ils sont reportés dans le journal L'Equipe du 16 Mai 1995 (p. 10) : « (...) même après un match remporté 73-6, comme ce fut le cas contre les Samoa, je peux trouver des critiques à émettre et les joueurs sont réceptifs. Oublier le tableau d'affichage, c'est gagner du temps pour travailler. " Enfin, une perspective positive subordonnée aux efforts à fournir sera évoquée en insistant sur le fait que les carences constatées peuvent être comblées lors des prochains entraînements.

\section{3 - Le développement et l'entretien d'un climat motivationnel}

D'après le théorie de Nicholls, une personne poursuivant des buts exclusivement orientés vers l'ego aura tendance à mettre en œuvre des stratégies destinées à recueillir des jugements favorables ou à échapper aux jugements défavorables des autres. Un sportif sûr de sa compétence n'est pas affecté par une telle orientation. En revanche, chez un sportif qui doute de son habileté, cela peut se traduire par des engagements dans des compétitions de moindre niveau où il est facile de briller ou bien encore par des abandons face à la difficulté. Le rôle des orientations d'accomplissement (tâche versus ego) des sportifs apparaît donc déterminant dans l'adoption des comportements d'assiduité ou d'abandon. Ces orientations subissent l'influence de la compétition, laquelle contribue à développer chez les individus des buts orientés vers l'ego. Par la comparaison sociale qu'elle instaure, elle constitue une arme à double tranchant jouant le rôle d'un outil motivationnel au service des seuls gagnants. Si pour Locke et Latham (1985) le contexte compétitif améliore la performance humaine, le sportif le mieux préparé et le moins perturbé par la comparaison sociale sera probablement celui qui parallèlement à des buts de compétition ambitieux poursuivra des buts de maîtrise également élevés. Rappelons, en effet, que ces deux types de buts ne sont pas incompatibles (Nicholls, 1984a, 1989, 1992) et que l'adoption de buts de compétition en sport n'hypothèque pas forcément la poursuite de buts de maîtrise (Gernigon \& Le Bars, 1995 ; Roberts, 1992 ; Roberts et al., 1996). Ceci a également été confirmé dans le cadre de l'étude concernant l'acquisition d'une habileté aquatique en situation dyadique (Gernigon, d'Arripe-Longueville, \& Huet, 1996). Le fait que les garçons aient développé des buts d'implication de l'ego et d'hypercompétitivité de manière plus importante que les filles n'a pas entravé chez eux la poursuite de buts référés à des standards internes ou absolus. Plus encore, l'orientation vers les standards sociaux ou normatifs très marquée chez ces garçons n'a altéré ni leur persévérance ni leurs progrès puisque ceux-ci se sont avérés supérieurs à ceux des filles. En fait, il ressort de ces études qu'une orientation vers la compétition peut correspondre à une stratégie efficace pour certaines personnes (e.g., les garçons) à condition toutefois qu'une telle orientation ne s'opère pas au détriment de l'adoption de buts de maîtrise. Si dans le contexte 
sportif, l'athlète est inévitablement amené à se fixer des buts compétitifs, il importe aussi de se préoccuper d'entretenir une orientation vers les buts de maîtrise corollairement aux autres buts.

Nous avons déjà signalé que le contexte dans lequel un individu était amené à exercer son activité influençait les orientations motivationnelles de celui-ci (Ames, 1984 ; Ames \& Ames, 1984 ; Ames \& Archer, 1988). Epstein (1988) met en avant le rôle de plusieurs facteurs, les variables "TARGET" (type de Tâches, nature de l'Autorité, Récompenses, organisation des Groupes, modalités d'Evaluation, Temps accordé pour atteindre les objectifs), comme éléments constitutifs de la structure du contexte d'accomplissement. Si ces auteurs se sont intéressés au domaine scolaire, Ames (1992) considère applicable au domaine de l'entraînement sportif l'intervention pédagogique sur ces facteurs dans le but d'instaurer un climat de maîtrise. Aussi, propose-t-elle d'agir sur les facteurs identifiés par Epstein :

\section{Type de tâche}

Proposer au sportif des tâches variées qui sollicitent des enjeux personnels (tâches individualisées) ainsi que son implication active. L'aider à se fixer des objectifs réalistes et accessibles à court terme.

\section{Autorité}

Faire participer le sportif à certaines décisions, lui confier des responsabilités vis-à-vis des autres. L'aider à développer des compétences pour s'auto-organiser et s'auto-évaluer.

\section{Reconnaissance}

Valoriser les qualités personnelles de chacun, les progrès individuels. Veiller à l'équité dans l'intérêt et les gratifications accordées à chaque membre de l'équipe.

\section{Groupements}

Adopter des formes de groupement variées et flexibles. Ne pas craindre d'utiliser l'hétérogénéité et notamment la diversité des compétences des individus.

\section{Evaluation}

Adopter une évaluation individualisée et signifiante pour chacun en prenant en compte des critères de progrès personnels et des critères de maîtrise en complément des critères de performance en compétition. Impliquer l'athlète dans les procédures d'évaluation de ses propres acquisitions (auto-évaluation).

\section{Temps}

Laisser aux sportifs le temps nécessaire pour qu'ils puissent s'améliorer. Les aider à organiser eux-mêmes leur travail et leur plan de progression. 
En fait, ces recommandations constituent un plaidoyer pour un climat d'entraînement démocratique contribuant à une éducation des attitudes de responsabilité et d'autodétermination. Loin d'être nié, le rôle de l'entraîneur doit plutôt être considéré ici comme organisateur d'une préparation à la performance personnalisée plus signifiante pour chaque athlète en ce qui concerne ses perceptions de contrôle et de compétence vis-à-vis d'objectifs à atteindre clairement identifiés. De plus, le rôle d'un type de climat particulier doit être envisagé en tenant compte de la perception de ce climat par l'individu. C'est en effet la manière dont ce climat est perçu et subjectivement conçu qui semble prédictive des cognitions responsables de l'adoption des comportements adaptatifs ou non. On ne peut donc que souligner la nécessité d'adapter le contexte d'entraînement aux caractéristiques des sportifs. Ainsi, pour Weinberg (1992), un des problèmes majeurs à résoudre est l'identification des buts les mieux appropriés à des personnes aux personnalités et aux styles motivationnels différents. Des études complémentaires mériteraient d'être entreprises afin d'appréhender les conditions et les modalités d'individualisation du climat motivationnel.

\section{CONCLUSION}

A la lecture de ce chapitre, l'entraîneur ou le sportif s'attendant à une forte valeur prescriptive des théories de la motivation dans le contexte sportif peut ressentir une certaine déception. Le fait que les perspectives d'intervention sur la motivation des sportifs, telles qu'elles ont été exposées, semblent limitées tient à plusieurs raisons. Tout d'abord, les sciences socio-cognitives, et en général, la psychologie ne sont pas des sciences exactes (si toutefois il en existe). La complexité des cognitions impliquées dans le comportement n'a d'égal que la multiplicité de interactions de ses déterminants. De plus, si certains de ces déterminants constituent actuellement des objets d'étude privilégiés, nombre d'entre eux restent encore à explorer ou à aborder dans le contexte du sport.

Quoiqu'il en soit, les limites à la prescriptibilité des modèles théoriques de la motivation à la préparation mentale de l'athlète sont celles de toute action humaine visant à agir sur le vouloir de l'humain. Par le passé, la psychologie cognitive imprégnée de la métaphore informatique a souvent considéré de manière réductrice le comportement comme une réponse résultant de processus calculatoires. Depuis quelques années cette psychologie s'intéresse également aux valeurs sociales qui guident les conduites de manière différenciée selon les individus. Devenant ainsi psychologie socio-cognitive, notamment à partir du développement des études sur la motivation d'accomplissement dans les années soixante dix, elle appréhende aussi les émotions générées par les perspectives et les constats d'atteinte ou de non atteinte de ces valeurs sociales. En s'intéressant à une complexité qui l'amène à sortir de plus en plus du laboratoire, elle ne peut plus faire l'économie de considérations humanistes trop souvent perçues comme l'exclusivité d'une psychologie thérapeutique ou prophylactique. 
En ce qui concerne la préparation physique du sportif, les transformations physiologiques relèvent d'une sollicitation intense, longue et régulière des substrats biologiques concernés. Il en va de même pour la préparation mentale conçue comme un long processus d'éducation ayant parmi ses buts la transformation et le développement des attitudes motivationnelles les mieux à même de développer chez un sportif les sentiments de contrôle, d'autodétermination et de compétence. S'interdisant de constituer un recueil de recettes applicables à tout sportif (ce qui aurait été certainement très séduisant pour l'usager de cet ouvrage, mais ô combien inefficace et illusoire!), les quelques recommandations qui précèdent ne se veulent être qu'un ensemble de repères pour celui qui veut seulement et modestement aider un athlète à se donner les moyens de ses ambitions. Puissent les propos de J. R. Germont (1995), responsable de la préparation olympique pour les jeux d'Atlanta illustrer la philosophie de la présente contribution : "Le bon entraîneur est celui qui fait tout pour ne pas être indispensable ».

\section{RÉFÉRENCES}

Abramson, L.Y., Seligman, M.E.P., \& Teasdale, J.D. (1978). Learned helplessness in humans: critique and reformulation. Journal of Abnormal Psychology, 87 (1), 49-74.

Alloy, L.B., Peterson, C.R., Abramson, L.Y., \& Seligman, M.E.P. (1984). Attributional style and the generality of learned helplessness. Journal of Personality and Social Psychology, 46, 681-687.

Ames, C. (1984). Achievement attributions and self-instructions under competitive and individual goal structures. Journal of Educational Psychology, 76, 478-487.

Ames, C. (1992). Achievement goals, motivational climate, and motivational processes. In G.C. Roberts (Ed.), Motivation in sport and exercise (pp. 161-176). Champaign, IL: Human Kinetics.

Ames, C., \& Ames, R. (1984). Systems of student and teacher motivation: Towards a qualitative definition. Journal of Educational Psychology, 76, 4, 535-556.

Ames, C., \& Archer, J. (1988). Achievement goals in the classroom: Students' learning strategies and motivation processes. Journal of Educational Psychology, 80, 260-267.

Atkinson, J.W. (1957). Motivational determinants of risk-taking behaviors. Psychological Review, 64, 359-372.

Bandura, A. (1977). Self efficacy: Toward a unifying theory of behavioral change. Psychological Review, 84, 191-215.

Bandura, A. (1997). Self-efficacy: The exercise of control. New York: Freeman.

Brière, N.M., Vallerand, R.J., Blais, M.R., \& Pelletier, L.G. (1995). Développement et validation d'une mesure de motivation intrinsèque, extrinsèque et d'amotivation en 
contexte sportif: L'Échelle de Motivation dans les Sports (ÉMS). International Journal of Sport Psychology, 26, 465-489.

Burton, D. (1989). Winning isn't everything: Examining the impact of performance goals on collegiate swimmers' cognitions and performance. The Sport Psychologist, 3, 105132.

Calder, B.J., \& Staw, B.M. (1975). Self-perception of intrinsic and extrinsic motivation. Journal of Personality and Social Psychology, 31, 599-605.

Carver, C.S., \& Scheier, M.F. (1981). Attention and self-regulation. New York: SpringerVerlag.

Csikszentmihalyi, M. (1975). Beyond boredom and anxiety. San Francisco: Josey-Bass.

Csikszentmihalyi, M. (1990). Flow: The psychology of optimal experience. New York: Harper \& Row.

De Charms, R. (1968). Personnal causation: The internal affective determinant. New York: Academic Press.

Deci, E.L. (1975). Intrinsic motivation. New-York: Plenum.

Deci, E.L., \& Ryan, R.M. (1980). Self-determination theory: When mind mediates behavior. Journal of Mind and Behavior, 1, 33-43.

Deci, E.L., \& Ryan, R.M. (1985). Intrinsic motivation and self-determination in human behavior. New York: Plenum Press.

Deschamps, J.C. (1987). Attribution et explication. In J.L. Beauveois, R.V. Joule et J.M. Monteil (Eds.), Perspectives cognitives et conduites sociales, tome 1. Cousset, Suisse: Del Val.

Duda, J.L. (1985). Goals and achievement orientations of Anglo and Mexican-American adolescents in sport and the classroom. International Journal of Intercultural Relations, 9, 131-155.

Duda, J.L. (1986a). Perceptions of sport success and failure among white, black, and hispanic adolescents. In J. Watkins, T. Reilly, \& L. Burwitz (Eds.), Sport science (pp. 214-222). London: E. \& F.N. Spon.

Duda, J.L. (1986b). A cross-cultural analysis of achievement motivation in sport and the classroom. In L. Vander Velden \& J. Humphrey (Eds.), Current selected research in the psychology and sociology of sport (pp. 117-131). New York: AMS Press.

Duda, J.L. (1988). The relationship between goal perspectives, persistence, and behavioral intensity among male and female recreational participants. Leisure Sciences, 10, 95106.

Duda, J.L. (1992). Motivation in sport settings: A goal perspective approach. In G.C. Roberts (Ed.), Motivation in sport and exercise (pp. 57-91). Champaign, IL: Human Kinetics. 
Duda, J.L. (1993). Goals: a social cognitive approach to the study of achievement motivation in sport. In R.N. Singer, M. Murphey \& L.K. Tennant (Eds.), Handbook of research on sport psychology (pp. 421-436). New York: Macmillan.

Duda, J.L., \& Allison, M.T. (1990). Cross-cultural analysis in exercise and sport psychology: A void in the field. Journal of Sport and Exercise Psychology, 12, 114131.

Duda, J.L., Newton, M., \& Chi, L. (1990). The relationship of task and ego orientation and expectations to multidimensional state anxiety. Paper presented at the annual meeting of the North American Society for the Psychology of Sport and Physical Activity, University of Houston, TX.

Durand, M. (1987). L'enfant et le sport. Paris: PUF.

Duval, S., \& Wicklund, R.A. (1972). A theory of objective self-awareness. New York: Academic Press.

Dweck, C.S. (1980). Learned helplessness in sport. In C.H. Nadeau, W.R. Halliwell, K.M. Newell, \& G.C. Roberts (Eds.), Psychology of motor behavior and sport - 1979 (pp. 1-11). Champaign, IL: Human Kinetics.

Dweck, C.S. (1986). Motivational processes affecting learning. American Psychologist, 41 (10), 1040-1048.

Elig, T.W., \& Frieze, I.H. (1979). Measuring causal attributions for success and failure. Journal of Personality and Social Psychology, 37, 621-634.

Epstein, J. (1988). Effective schools or effective students ? Dealing with diversity. In R. Haskins \& B. MacRae (Eds.), Policies for America's Public Shools (pp. 89-126). Norwood, NJ: Ablex.

Germont, J.R. (Septembre 1995). La préparation mentale au geste sportif. Communication au Colloque Eurathlon : l'entraînement mental et la performance sportive de haut niveau, Paris, INSEP - Réseau Européen de Instituts des Sciences du Sport .

Gernigon, C. (1996). Approche cognitive de la régulation de la motivation. In H. Hélal, E. Jousselin \& Y. Demarais (Eds), La récupération en sport: Approches des techniques et des moyens. Les cahiers de l'INSEP, $n^{\circ} 14-15$ (pp. 75-81). Paris: INSEP Publications.

Gernigon, C. (1998). Fixer des buts pour l'entraînement et pour la compétition. In E. Thill et P. Fleurance (Eds.), Guide pratique de psychologie du sport. Paris: Vigot.

Gernigon, C., Arripe-Longueville, F. d', \& Huet, M.L. (1996). Sexe, buts personnels, expectations d'efficacité, persévérance et apprentissage d'une habileté motrice en situation dyadique. Journal International de Psychologie, 31 (3,4), 289.

Gernigon, C., \& Le Bars, H. (1995). Effects of sport practice and gender on children's and adults' achievement goals in aïkido and judo. In R. Vanfraechem-Raway \& Y. Vanden Auweele (Eds.), Proceedings of the IXth European Congress on Sport Psychology, Part I (pp. 331-338). Bruxelles: FEPSAC. 
Gill, D.L. (1986). Competitiveness among females and males in physical activity classes. Sex Roles, 15, 233-247.

Gill, D.L. (1988). Gender differences in competitive orientation and sport participation. International Journal of Sport Psychology, 19, 145-159.

Griffin, N.S., \& Keogh, J.F. (1981). Movement confidence and effective movement behavior in adapted physical education. Motor skills: Theory into practice, 5, 23-25.

Griffin, N.S., \& Keogh, J.F. (1982). A model for movement confidence, in J.A. Kelso \& J.E. Clark (Eds.), The development of movement control and coordination. New York: Wiley.

Guthrie, E.R. (1957). The psychology of learning. New York: Harper \& Brothers.

Hall, H.K., \& Kerr, A.W. (1997). Motivational antecedents of precompetitive anxiety in youth sport. The Sport Psychologist, 11 (1), 24-42.

Hanrahan J.S., \& Grove J.R. (1990). Further examination of the psychometric properties of the sport attributional style scale. Journal of Sport Behavior, 13 (4) 183-193.

Hanrahan J.S., Grove J.R., \& Hattie, J.A. (1989). Development of a questionnaire measure of sport related attributional style. International Journal of Sport Psychology, 20 (2), 114-134.

Harter, S. (1978). Effectance motivation reconsidered: Toward a developmental model. Human Development, 21, 34-64.

Harter, S. (1982). The perceived competence scale for children. Child Development, 53, 87-97.

Harter, S. (1990). Causes, correlates, and the functional role of global self-worth: A lifespan perspective. In R.J. Sternberg \& J. Kolligian (Eds.), Competence considered (pp.67-97). New Haven: Yale University Press.

Heckhausen, H. (1974). Leistung und chancengleichheit. Göttingen, Germany: Hogrefe.

Heider, F. (1958). The psychology of interpersonal relations. New York: J. Wiley \& Sons.

Hiroto, D.S. (1974). Locus of control and learned helplessness. Journal of Experimental Psychology, 102 (2), 187-193.

Hiroto, D.S., \& Seligman, M.E.P. (1975). Generality of learned helplessness in man. Journal of Personality and Social Psychology, 31 (2), 311-327.

Horn, T.S. (1987). The influence of teacher coach-behavior on the psychological development of children. In D. Gould \& M.E. Weiss (Eds.), Advances in pediatric sport sciences: Vol. 2. Behavioral issues (pp. 121-142). Champaign, IL: Human Kinetics.

Jackson S.A. (1995). Factors influencing the occurrence of flow state in elite athletes. Journal of Applied Sport Psychology, 7, 138-166. 
Jones, E.E., \& Davis, K.E. (1965). From acts to dispositions: The attribution process in person perception. In L. Berkowitz (Ed.), Advances in experimental social psychology, Vol. 2 (pp. 219-266). New York: Academic Press.

Kavussanu, M., \& Roberts, G.C. (1996). Motivation in physical activity contexts: The relationship of perceived motivational climate to intrinsic motivation and selfefficacy. Journal of Sport and Exercise Psychology, 18, 264-280.

Kelley, H.H. (1967). Attribution theory in social psychology. In D. Levine (Ed.), Nebraska Symposium of Motivation (pp. 192-238). Lincoln: University of Nebraska Press.

Kukla, A. (1972). Foundations of an attributional theory of performance. Psychological Review, 79, 454-470.

Kyllo, L.B. \& Landers, D.M. (1995). Goal setting in sport and exercise: A research synthesis to resolve the controversy. Journal of Sport and Exercise Psychology, 17, 117-137.

Langer, E.J. (1975). The illusion of control. Journal of Personality and Social Psychology, 32, 311-328.

Lepper, M.R., Greene, D., \& Nisbett, R.E. (1973). Undermining children's intrinsic interest with extrinsic rewards: A test of the overjustification hypothesis. Journal of Personality and Social Psychology, 28, 129-137.

Locke, E.A. (1991). Problems with goal-setting research in sports - and their solution. Journal of Sport and Exercise Psychology, 13, 311-316.

Locke, E.A., \& Latham, G.P. (1985). The application of goal setting to sports. Journal of Sport Psychology, 7, 205-222.

Maehr, M.L. (1984). Meaning and motivation: Toward a theory of personal investment. In R. Ames \& C. Ames (Eds.), Research on motivation in education, Vol. 1: Student motivation (pp.115-144). Orlando, FL: Academic Press.

Martens, R. (1977). Sport Competition Anxiety Test. Champaign, IL: Human Kinetics.

Martens, R., Vealey, R.S., \& Burton, D. (Eds.) (1990). Competitive anxiety in sport. Champaign, IL: Human Kinetics.

McClelland, D.C., Atkinson, J.W., Clark, R.W., \& Lowell, E.L. (1953). The achievement motive. New York: Appleton-Century-Crofts.

Miller, I.W., \& Norman, W.H. (1979). Learned helplessness in humans: A review and attribution theory model. Psychological Bulletin, 86, 93-118.

Mouanda, J.(1989). Etude de la motivation intrinsèque en situation de hand-ball. Revue STAPS, 10, (19), 72-74.

Mucchielli, A. (1994). La psychologie sociale. Paris: Hachette.

Murray, H.A. (1938). Explorations in personality. New York: Oxford University Press. 
Nicholls, J.G. (1978). The development of the concepts of effort and ability, perception of academic attainment, and the understanding that difficult tasks require more ability. Child Development, 49, 800-814.

Nicholls, J.G. (1984a). Achievement motivation: Conceptions of ability, subjective experience, task choice, and performance. Psychological Review, 91 (3), 328-346.

Nicholls, J.G. (1984b). Conceptions of ability and achievement motivation. In R. Ames \& C. Ames (Eds.), Research on motivation in education, Vol. 1 (pp. 39-73). New York: Academic Press.

Nicholls, J.G. (1989). The competitive ethos and democratic education. Cambridge, MA: Harvard University Press.

Nicholls, J.G. (1992). The general and the specific in the development and expression of achievement motivation. In G.C. Roberts (Ed.), Motivation in sport and exercise (pp. 31-56). Champaign, IL: Human Kinetics.

Nicholls, J.G., \& Miller, A.T. (1984). Development and its discontents: The differentiation of the concept of ability. In J.G. Nicholls (Ed.), Advances in motivation and achievement, Vol. 3: The development of achievement motivation (pp. 185-218). Greenwich, CT: JAI Press.

Nuttin, J. (1985). Théorie de la motivation humaine. Paris: PUF (2 éd.).

Orlick, T.D., \& Mosher, R. (1978). Extrinsic rewards and participant motivation in a sport related task. International Journal of Sport Psychology, 9, 27-39.

Peterson, C., Maier, S.F., \& Seligman, M.E.P. (1993). Learned helplessness: A theory for the age of personal control. New York: Oxford University Press.

Peterson, C., \& Seligman, M.E.P. (1984). Causal explanations as a risk factor for depression: Theory and evidence. Psychological Review, 91 (3), 347-374.

Peterson, C., Semmel, A., Von Bayer, C., Abramson, L.Y., Metalsky, G.I., \& Seligman, M.E.P. (1982). The attributional style questionnaire. Cognitive Therapy and Research, 6, 287-299.

Prapavessis, H., \& Carron, A.V. (1988). Learned helplessness in sport. The Sport Psychologist, 2, 189-201.

Rettew, D.C., Reivitch, K., Peterson, C., Seligman, D.A., \& Seligman, M.E.P. (1990). Professional baseball, basketball, and explanatory style: Predicting performance in the major leagues. Manuscript, University of Pennsylvania.

Roberts, G.C. (1984). Toward a new theory of motivation in sport: The role of perceived ability. In J.M. Silva and R.S. Weinberg (Eds.), Psychological foundations of sport. Champaign, IL: Human Kinetics.

Roberts, G.C. (1992). Motivation in sport and exercise: Conceptual constraints and convergence. In G.C. Roberts (Ed.), Motivation in sport and exercise (pp. 3-29). Champaign, IL: Human Kinetics. 
Roberts, G.C., \& Treasure, D.C. (1994). Sport de compétition et motivation chez l'enfant: Approche à partir de la théorie des buts d'accomplissement. In Sport et développement de l'enfant, Revue Enfance, 2-3, 161-169.

Roberts, G.C., Treasure, D.C., \& Hall, H. K. (1994). Parental goal orientations and beliefs about the competitive-sport experience of their child. Journal of Applied Social Psychology, 24 (7), 631-645.

Roberts, G.C., Treasure, D.C., \& Kavussanu M. (1996). Orthogonality of achievement goals and its relationship to beliefs about success and satisfaction in sport. The sport psychologist, 10, 398-408.

Ross, L. (1977). The intuitive psychologist and his shortcomings: Distorsions in the attribution process. In L. Berkowitz (Ed.), Advances in experimental social psychology, vol. 10. New York: Academic Press.

Roth, S. (1980). A revised model of learned helplessness in humans. Journal of Personality, 48, 103-133.

Ryan, E.D. (1980). Attribution, intrinsic motivation, and athletics: A replication and extension. In C.H. Nadeau, W.R. Halliwell, K.M. Newell, \& G.C. Roberts (Eds.), Psychology of motor behavior and sport - 1979 (pp. 19-26). Champaign, IL: Human Kinetics.

Sanders, A.F.(1983). Towards a model of stress and human performance. Acta Psychologica, 53, 61-97.

Seligman, M.E.P. (1975). Helplessness: On depression, development, and death. San Francisco: Freeman.

Seligman, M.E.P. (1990). Learned optimism. New York: Pocket Books.

Seligman, M.E.P., Maier, S.F., \& Geer, J.H. (1968). Alleviation of learned helplessness in the dog. Journal of Abnormal Psychology, 73 (3), 256-262.

Seligman, M.E.P., Nolen-Hoeksema, S., Thornton, N., \& Thornton, K.M. (1990). Explanatory style as a mechanism of disappointing athletic performance. Psychological Science, 1, 143-146.

Seligman, M.E.P., \& Peterson, C. (1986). Explanatory style of NBA players. Unpublished data, University of Pennsylvania.

Stipek, D.J. (1993). Motivation to learn, from theory to practice. Boston: Allyn \& Bacon (2nd ed.).

Sweeney, P.D., Anderson, K., \& Bailey, S. (1986). Attribution style in depression: A meta-analytic review. Journal of Personality and Social Psychology, 50, 974-991.

Theeboom, M., De Knop, P., \& Weiss, M.R. (1995). Motivational climate, psychological responses, and motor skill development in children's sport: A field-based intervention study. Journal of Sport and Exercise Psychology, 17, 294-311. 
Thill, E. (1987). Préparations psychologiques alternatives à la performance: L'utilisation de rituels ou la systématisation de moyens d'autocontrôle comportemental. Conférence au IVe Congrès International de Psychologie du Sport, Bruxelles.

Thill, E. (1989). Motivation et stratégies de motivation en milieu sportif. Paris: PUF.

Thill, E., \& Mouanda, J. (1990). Autonomie ou contrôle en contexte sportif: Validité de la théorie de l'évaluation cognitive. International Journal of Sport Psychology, 21, 1-20.

Thomas, J.R. \& Tennant, L.K. (1978). Effects of rewards on children's motivation for an athletic task. In F. Smoll \& R. Smith (Eds.), Psychological perspectives in youth sports. Washington, DC: Hemisphere.

Vealey, R.S.,\& Campbell, J.L. (1988). Achievement goals of adolescent figure skaters: Impact on self-confidence, anxiety, and performance. Journal of Adolescent Research, 3, 227-243.

Walling, M.D., Duda, J.L., \& Chi, L. (1993). The perceived motivational climate in sport questionnaire: Construct and predictive validity. Journal of Sport and Exercise Psychology, 15, 172-183.

Weinberg, R.S. (1992). Goal-setting and motor performance: A review and critique. In G.C. Roberts (Ed.), Motivation in sport and exercise (pp. 177-197). Champaign, IL: Human Kinetics.

Weinberg, R.S., \& Gould, D. (1995). Foundations of sport and exercise psychology. Champaign, IL: Human Kinetics.

Weinberg, R.S., \& Weigand, D.A. (1993). Goal setting in sport and exercise: A reaction to Locke. Journal of Sport and Exercise Psychology, 15, 88-96.

Weinberg, R.S., \& Weigand, D.A. (1996). Let the discussions continue: A reaction to Locke's comments on Weinberg and Weigand. Journal of Sport and Exercise Psychology, 18, 89-93.

Weiner, B. (1985). An attributional theory of achievement motivation and emotion. Psychological Review, 92 (4), 548-573.

Weiner, B. (1986). An attributional theory of motivation and emotion. New York: Springer-Verlag.

Weiss, M.R., \& Chaumeton, N. (1992). Motivational orientations in sport. In T.S. Horn (Ed.), Advances in sport psychology (pp. 61-99). Champaign, IL: Human Kinetics.

White, R.W. (1959). Motivation reconsidered: The concept of competence. Psychological Review, 66, 297-333.

White, S.A., \& Duda, J.L. (1991). The interdependence between goal perspectives, psychological skills, and cognitive interference among elite skiers. Paper presented at the annual meeting of the Association for the Advancement of Applied Sport Psychology, Savannah, GA. 
White, S.A., \& Duda, J.L. (1994). The relationship of gender, level of sport involvement, and participation motivation to task and ego orientation. International Journal of Sport Psychology, 25, 4-18. 